\title{
Impact of lightweight and conventional jackhammers on the operator
}

\author{
Naira Campbell-Kyureghyan, $\mathrm{PhD}^{*}$, Gurjeet Singh, MS , Wilkistar Otieno, PhD, Karen Cooper, MS \\ Department of Industrial \&Manufacturing Engineering, University of Wisconsin-Milwaukee, Milwaukee, WI, USA
}

\begin{abstract}
Jackhammer manufacturers have recently developed lightweight (45-60 lbs) jackhammers intended to reduce the required lifting and pushing forces during operation. However, the vibration characteristics of the lightweight jackhammers and their effect on muscle activity are currently unknown. The objective of this study was to compare the measured vibration and muscle activity between: (i) conventional (90lb) and light weight (60lb) jackhammers, (ii) different pavement type/thickness combinations, and (iii) pneumatic and hydraulic jackhammers. Five jackhammers were tested on 4 and 6 inch thick asphalt and concrete pavements by four experienced operators. Analysis of the results revealed that both weight classes averaged $9.7 \mathrm{~m} / \mathrm{s}^{2}$ at the $20 \mathrm{~Hz}$ weighted $1 / 3$ octave band frequency, and the TLV of daily exposure for either weight class of jackhammer was less than 1.5 hours/per day. There was an approximately $33 \%$ difference in vibration measured on the hand of the operators due to pavement thickness, $30 \%$ due pavement type, and no difference due to power source. Conventional jackhammers overall produced higher muscle activity than lightweight jackhammers. Although selection of the correct jackhammer for the job involves many factors including pavement type and thickness, the results of this research can be used to assist in selecting the appropriate jackhammer.
\end{abstract}

Keywords: hand transmitted vibration, muscle activity, jackhammer, dynamic response, frequency weighting

\section{Introduction}

Jackhammers are commonly used in all sectors of the construction industry and range in size from small electric units to large, heavy units mounted on equipment. For hand-operated jackhammers there is often a trade-off between efficiency for the task and user comfort. To operate a $90 \mathrm{lb}$ jackhammer, an operator needs to be able to exert substantial force while continuously exposed to vibration.

ISO standards exist for exposure to hand-arm and whole body vibration exposure. The standards define allowable exposure time for different acceleration levels. Dong, et al. [2,3] investigated the effects of vibration on human discomfort using accelerometers and pressure sensing mats. The research was aimed at improving standards for human exposure to vibration, and indicated that the allowable limits may be nonconservative in the higher frequency ranges.

Hartung et al. [4] investigated grip and push force and muscle activity while using vibrating power tools.
The test protocol consisted of 8 minute exposures with 6 minute rest breaks between trials, and the researchers monitored muscle activity during both the trial and the rest break. Muscle activity was found to increase with exposure to vibration. During the rest period the measured effects of the vibration decreased, and by the end of the rest period was close to, but still above, the pre-test responses.

The prevalence of hand-arm vibration syndrome among jackhammer and blaster operators was investigated by Dasgupta et al. [1]. The study found that neuroplasty and musculoskeletal abnormities were associated with the symptoms, rather than peripheral circulation disorders.

In summary, previous literature reported that muscle activity was amplified with increased exposure to vibration [4], and could lead to a $40 \%$ higher chance of developing symptoms of soft tissue wasting in the hands among conventional jackhammer operators [1].

Jackhammer manufacturers have recently developed lightweight (45-60 lbs) jackhammers intended

\footnotetext{
*Corresponding author - E-mail: campbeln@uwm.edu -Mail: P.O. Box 784, Milwaukee, WI, 53201- Ph: 4142293403
} 
to reduce the required lifting and pushing forces during operation. However, the vibration characteristics of the lightweight jackhammers and their effect on muscle activity are currently unknown.

The objectives of this study were to compare the measured vibration and muscle activity between: (i) conventional (90lb) and light weight (60lb) jackhammers, (ii) different pavement type/thickness combinations, and (iii) pneumatic and hydraulic (601b \& 901b) jackhammers.

\section{Methods}

\subsection{Experimental design}

The testing protocol was designed to simulate an actual working environment. Each trial consisted of breaking up a $3 \times 3$ foot square section of pavement. Five diagonal lines were evenly spaced across the square as a guide on how the pavement should be broken up. Two pavement type, asphalt and concrete, and two pavement thicknesses, four and six inches, were used in the tests. Five jackhammers (2 conventional and 3 lightweight) were used in this study. Three of the jackhammers were pneumatically powered, while the other two were hydraulic. Thus, each jackhammer was used in four trials by each operator, for a total of 20 experimental conditions per operator. A rest period of 15 minutes was allowed between trials.

\subsection{Subjects}

Four experienced operators participated in each of the experimental conditions described above, for a total of 80 randomized trials. The operators were all males, averaged 36 years of age and 66.5 inches tall, and weighed between $150-180 \mathrm{lbs}$. The years of experience working with the jackhammer ranged from 1-13 years.

Each operator completed the trials in a random order, starting with a different combination of jackhammer, pavement type and pavement thickness. Personal protective equipment (PPE) as recommended by the jackhammer manufacturers was worn in all trials. Each day of testing consisted of two sessions, one in the morning and one in the afternoon.

\subsection{Instrumentation}

A single high frequency accelerometer (NexGen Ergonomics, CA) was attached to the left handle of the jackhammer to measure the vibration produced by the jackhammer. Three wireless $3 \mathrm{D}$ accelerometers (DelSys TRIGNO, USA) were placed on the left and right forearm, and the left hand of the operator. The right hand was not instrumented with an accelerometer since a pressure-sensing glove was placed on that hand. Twelve wireless electromyography (EMG) sensors were used to collect muscle activity on each operator during each trial for 6 muscle pairs: (Table 1). Electrodes were placed according to Cram et al. (1998).

\subsection{Analysis}

The RMS of the vibration was calculated using a sliding window with a size of 512 data points. A $2^{\text {nd }}$ order Butterworth filter with a low pass filter of $4 \mathrm{~Hz}$ was applied to the data. A Fast Fourier Transform was performed and the data was organized in $1 / 3$ octave bands. Frequency weight factors, as defined in ISO 5349, were applied to the data to obtain the weighted $1 / 3$ octave bands. This data was then compared to ISO standard 5349 and to the Threshold Limit Values (TLV).

A custom MATLAB program was written to analyze the data collected by the 12 TRIGNO wireless EMG sensors. The program calculated the RMS value for each sensor at each trial as well as the location and magnitude of the top 10 peaks for each sensor. The difference between weight classes of jackhammer was determined by finding the ratio of RMS values between a conventional and lightweight jackhammer while maintaining the same power source. Similarly, the difference between power sources was determined by finding the ratio of RMS values between hydraulic and pneumatic jackhammers while maintaining weight.

\subsection{Statistical analysis}

The data was statistically analyzed to determine the most significant source(s) of variability. Two types of models were used in the analysis namely, the generalized linear models (GLM) for factorial designs and a 4-stage nested mixed model, where the jack hammer weight and power source were treated as fixed factors while the subjects, pavement type and thickness we treated as random factors. The data was categorized by jack hummer weight $(60 \mathrm{lbs}$ and $90 \mathrm{lbs}$ ) and by power source (pneumatic and hydraulic) in the nested design model. All the analyses were carried out at 0.05 significance levels, but those 
instances in which some factors that were significant at 0.1 level of significance are also reported.

\section{Results}

There were some differences observed in vibration measured on the handle between jackhammers. Conventional weight jackhammers exhibited an average peak acceleration of $4.2 \mathrm{~m} / \mathrm{s}^{2}$ in the $25 \mathrm{~Hz}$ weighted $1 / 3$ octave band frequency along the direction of the forearm, while lightweight jackhammers had an average peak acceleration of $4.0 \mathrm{~m} / \mathrm{s}^{2}$ in the same frequency band. A statistically significant difference $(p=0.007)$ was seen in handle vibration between hydraulic and pneumatic jackhammers, with average peak accelerations of 2.9 and $4.1 \mathrm{~m} / \mathrm{s}^{2}$ respectively in the $31.5 \mathrm{~Hz}$ weighted $1 / 3$ octave band frequency. Additionally, pavement thickness was found to be a statistically significant factor $(p=0.001)$ affecting handle vibration for both grouping by power source and by weight. However, both jackhammer weight classes and power sources fall within the 4-8 hour allowable exposure range according to ISO 5349.

When considering vibrations measured on the hand, little difference was observed between jackhammer categories, but some differences were seen based on pavement characteristics. Pavement thickness significantly $(p<0.05)$ affected the results for all groupings, while pavement type (asphalt vs. concrete) made a significant difference $(p<0.05)$ only for pneumatic jackhammers. The Threshold Limit Values are a single number for evaluating the allowable exposure due to vibration, based on the weighted RMS accelerations (aRMS). Both weight classes had an aRMS of $9.7 \mathrm{~m} / \mathrm{s}^{2}$, and the Threshold Limit Value (TLV) of daily exposure for either weight class of jackhammer was less than 1.5 hours per day (Figure $1)$. There is a slight difference $(p=0.002)$ in aRMS due to power source (Figure 2), with pneumatic jackhammers recording a slightly higher aRMS value $\left(9.9 \mathrm{~m} / \mathrm{s}^{2}\right.$ vs $\left.9.4 \mathrm{~m} / \mathrm{s}^{2}\right)$.

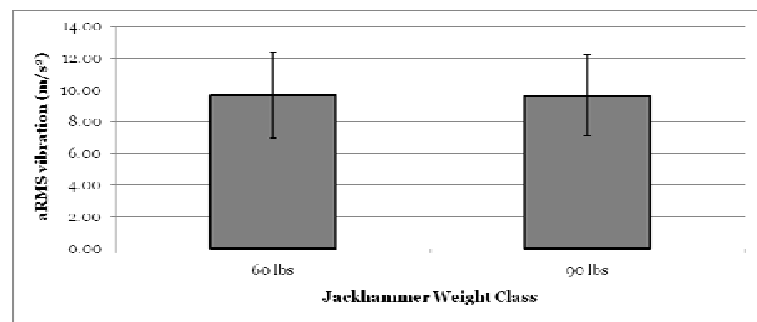

Fig 1: Comparison of aRMS measured on the operators hand by jackhammer weight class.

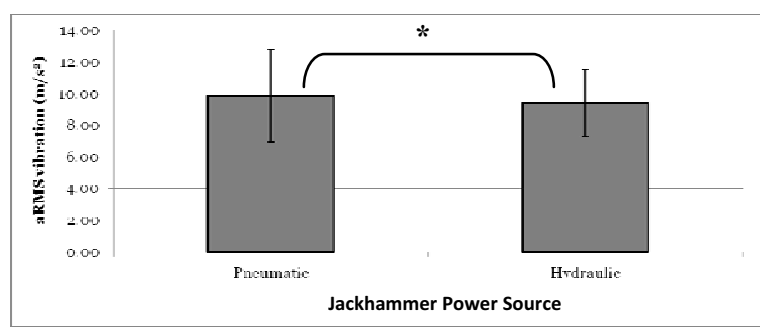

Fig 2: Comparison of aRMS measured on the operators hand by jackhammer power source. Significant differences with $p$-value less than 0.05 are denoted by an asterisk $\left(^{*}\right)$.

The difference in vibration between pavement types and thicknesses was evaluated using the Vibration Dose Value (VDV), which accounts for the duration of vibration. There was an approximately $30 \%$ increase $(p<0.05)$ in vibration measured on the operators' hand (Figure 3) when comparing asphalt to concrete pavement types. Similarly, there was an approximately $33 \%$ increase $(p<0.05)$ in vibration measured on the hand of the operators (Figure 4) when comparing 6 inch pavements to 4 inch pavements.

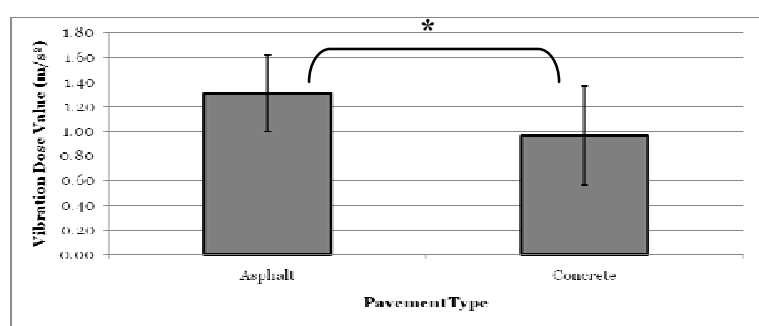

Fig 3: Comparison of vibrations measured on the operators hand by pavement type. Significant differences with $p$-value less than 0.05 are denoted by an asterisk $(*)$. 


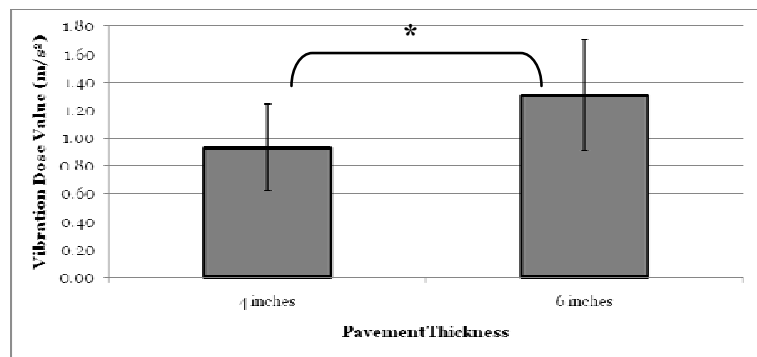

Fig 4: Comparison of vibrations measured on the operators hand by pavement thickness. Significant differences with $p$-value less than 0.05 are denoted by an asterisk $(*)$.

Conventional jackhammers produced higher muscle activity than lightweight jackhammers in all muscles except for the right trapezius (Table 1). Over two-fold difference in muscle activation was observed in the left bicep while operating conventional as compared to lightweight jackhammers. The trapezius and triceps are the only muscles to show consistent increase in bilateral muscle activity when using hydraulic jackhammers compared to pneumatic jackhammers.

\section{Discussion and summary}

This study investigated the effect on hand vibration and muscle activity of differences in jackhammer weight and power source. Overall, the jackhammer weight and power source were found to have very little effect on the vibration transmitted to the hand. Additionally, all jackhammers were limited in the duration for which it was safe to use them according to both ISO standards and the TLV. The pavement type and thickness did have a large affect on the vibration. This is to be expected since the interaction of the jackhammer and pavement will affect the vibration response.

Conventional jackhammers consistently required greater muscle activation than did lightweight jackhammers. The higher weight of the conventional jackhammers should require more force to move and control. Hydraulic jackhammers also generated more muscle activity than did pneumatic jackhammers. The hydraulic jackhammers tended to be more powerful, again suggesting that they would require high muscle forces to use.
Further analysis of the data found that pavement thickness had a somewhat significant $(p<0.1)$ effect on activation of the dominant arm muscles (right biceps and triceps). Observations indicated that thicker pavement required more lifting and repositioning of the jackhammers, and the task required a longer time. Both additional lifting and longer task times would lead to higher demands on the arm muscles. Pavement type was found to be a significant factor only at $\alpha=0.10$ for torso muscles (rectus abdominis and erector spinae) activation. One possible explanation is that asphalt pavements were more ductile, and the operators were observed to bend forward during pavement breaking to apply additional body weight to the jackhammer. Conversely, relatively brittle concrete did not require extra force to power the jackhammer through the pavement.

Overall, lightweight jackhammers had a lesser impact on the operator in terms of vibration and muscle activity, indicating that they could be safer to use. Although selection of the correct jackhammer for the job involves many factors including pavement type and thickness, the results of this research can be used to assist in selecting the appropriate jackhammer.

\section{Acknowledgement}

This project was sponsored by the Gas Technology Institute. Mr. Daniel Strobel assisted with data collection and processing.

\section{References}

[1] A. Dasgupta, J. Harrison, Effects of vibration on the hand-arm system of miners in India. Occup Med, 46(1996), 71-78.

[2] R.G. Dong, A.W. Schopper, T.W. McDowell, D.E. Welcome, J.Z. Wu, W.P. Smutz, C. Warren, S. Rakheja, Vibration energy absorption (VEA) in humanfingers-hand-arm system. Medical Engineering and Physics, 26 (2004a), 483-492.

[3] R. G. Dong, D. E. Welcome, T. W. McDowell, J. Z. Wu, Biodynamic Response of Human Fingers in a Power Grip Subjected to a Random Vibration, Journal of Biomechanical Egineering, 126 (2004b), 447-458.

[4] E. Hartung, H. Dupuis, M. Scheffer, Effects of grip and push forces on the acute response of the hand-arm system under vibrating conditions. Int Arch of Occup and Envir Health, 64 (1993), 463-467. 
Table 1: Ratio of average EMG between jackhammer weight (conventional/lightweight) and power source (hydraulic/pneumatic)

Conventional/Lightweight

\begin{tabular}{|c|c|c|c|c|}
\hline \multirow[b]{2}{*}{ Muscles } & \multicolumn{2}{|c|}{ - } & \multicolumn{2}{|c|}{ - } \\
\hline & EMG ratio (R) & EMG ratio (L) & EMG ratio (R) & EMG ratio (L) \\
\hline Bicep & 1.12 & 2.40 & 1.33 & 0.92 \\
\hline Tricep & 1.00 & 1.21 & 1.12 & 1.18 \\
\hline Deltoid & 1.22 & 1.16 & 1.07 & 1.00 \\
\hline Rectus Abdominis & 1.27 & 1.22 & 0.96 & 1.31 \\
\hline Erector Spinae & 1.12 & 1.07 & 1.10 & 1.09 \\
\hline Trapezius & 0.90 & 1.28 & 1.20 & 1.35 \\
\hline
\end{tabular}

